\title{
Anatomical and Surgical Basis for Adult Living Donor Liver Transplantation with the Right Liver Lobe
}

\author{
Borz C, Marian D, Bara T Jr, Jimborean O, Bara T, Márton D \\ Surgical Clinic 2, County Emergency Clinical hospital, Tîrgu Mureș, Romania
}

\begin{abstract}
Liver transplantation is now a standard procedure for the treatment of end stage liver diseases. Since 1968 until 2012, a number of 113,627 liver transplantations were performed in Europe, in 28 countries and 153 institutions. Despite these impressive figures the waiting list is growing every year. Transplant surgeons were preoccupied to find new ways to increase the donor pool. Among them: reduced size liver transplantation, split liver technique and more recently living donor liver transplantation. At first in the early '90, living donor liver transplantation was used for pediatric patients because the left lateral hepatic segments were harvested. This graft is too small for the metabolic demands of an adult patient. So the next step was the harvesting of the right liver lobe from the donor and transplantation to adult patients. Living donor liver transplantation has gained fast a wide acceptance but there are a few issues to discuss. The main concern is about the donor safety which is a healthy person undergoing major surgery with potential risks. Also the surgical technique evolved due to a better understanding of the anatomy and physiology of the liver and the right liver graft. We discuss here the anatomical and surgical basis for living donor liver transplantation with the right liver lobe.
\end{abstract}

Keywords: liver transplantation, living donor, right lobe

Received: 22 April 2014 / Accepted: 25 May 2014

\section{Introduction}

The first successful liver transplant was performed by Starzl in 1967 [1]. The introduction of cyclosporine in 1983 lead to a spectacular rise of the number of liver transplantations. Unfortunately however, the waiting list was also growing so that at a point the cadaveric liver donor pool was not enough anymore. Due to the scarcity of cadaveric livers, transplant surgeons started to introduce new techniques in order to provide a higher number of organs for transplantation. For instance, in 1984 Bismuth in Paris [1]introduced the reduced size liver transplantation to accommodate a graft in smaller patients or children, and Pichlmayr in 1988 in Hannover [1] used the split liver technique to obtain two grafts, for an adult (the larger part) and for a child (the smaller part). However, this was not enough to increase sufficiently the donor pool. In 1989 Raia in Sao Paulo [1] performed two liver transplantations using partial liver grafts (left lateral segment) from living donor, but the recipients died. The first successful living donor liver transplant was made by Strong in 1990 in Brisbane [1]. The recipient was a 15 months old Japanese child with biliary atresia. Strong was followed in the same year by $\mathrm{Na}$ gasue in Japan [1] and from this point the procedure was embraced in many transplantation centers, especially in Asian countries where on religious grounds the cadaveric transplantation was not possible. Pediatric recipients were the first to benefit from living donor liver transplantation, using the left lateral segments from the living donor. In the USA the first series of LDLT were performed by the team

Correspondence to: Dorin Marian

E-mail: dorinmar@rdslink.ro of Broelsch [1]. The effect of this procedure was a dramatic decrease on the waiting list in pediatric patients. In an effort made to expand the donor pool also in the adult patients, the utilization of the right liver lobe from the living donor was first introduced by Yamaoka and Ozawa [1] in 1993. Then, in 1995 Broelsch in Hamburg [1] proceeded with harvesting right liver grafts for adult patients. At first, right hepatectomy in living donor raised concerns about the great amount of liver resected from the donor $(60 \%)$ and was feared for postoperative liver failure in the donor. Development of the technique and the strategy in both donor and recipients made possible the spreading of the living donor liver transplantation all over the world.

\section{Hepatic mass regeneration. Physiological considerations}

The liver has the potential to regenerate after resection. When a small liver is transplanted to a larger recipient, it enlarges proportionally to the recipient. This hepatic regeneration is the key to the success of living donor liver transplantation. The lobes or segments transplanted in the recipient, as well as the portion of the remnant liver, return to normal size in about 30 days [2]. The minimal hepatic tissue needed to support a normal function appears to be $30 \%$ from the entire hepatic mass or $0.8 \mathrm{~g} / \mathrm{kg}$ of body weight. All-in-one Computer Tomography is a very useful volumetric tool for evaluating the hepatic mass and the size of the graft. Several centers use the graft to body weight ratio (GBWR) for the evaluation. To perform a successful living donor liver transplantation with the right lobe, the graft to body weight ratio needs to be more than $0.8 \%$ and in some centers at least 1\% [3]. Below this value there is a 
risk for hepatic failure, prolonged cholestasis and increased mortality of the recipient [4]. Another suggested formula is represented by graft weights as a percentage of standard liver mass. As a comparison, $50 \%$ of standard liver mass represents a graft to body weight ratio of $1 \%$. Urata [5] has calculated the standard liver volume optimal for the metabolic demands of the recipients as follows:

standard liver volume $(\mathrm{ml})=706.2 \times$ BSA (body surface area) $\left(\mathrm{m}^{2}\right)+2.4$ or

standard liver volume $(\mathrm{ml})=2.223 \times \mathrm{BW}$ (body weight) $(\mathrm{kg}) \times \mathrm{BH} 0.682$ (body height) $(\mathrm{cm})$.

The right lobe from a donor (segments V, VI, VII, VIII) represents $60 \%$ of the liver mass. The average weight of a right lobe graft is $875 \mathrm{~g}$.

Steatosis is a risk factor for primary non-function of the transplanted liver, reducing the percentage of functioning liver and also diminishing hepatic regeneration. It is important to perform the liver biopsy in order to assess the degree of steatosis. Calculation of graft to body weight ratio must be done in relation with steatosis. Each percentage of fat reduces the functional hepatic mass of the graft by $1 \%$. Recent studies showed a correlation of steatosis with BMI (body mass index). Persons with BMI less than 25 seldom have steatosis. Liver biopsy is also important to exclude rare diseases of the donor liver in order not to compromise the donor operation outcome.

\section{Surgical technique}

The donor operation is the key of the procedure because we have to follow the principle: primum non nocere (firstly do not harm). The donor is a healthy person and the operation must not jeopardize his/her safety. Before the procedure the donors are asked to auto-donate 2 units of blood. The surgical technique was refined due to advances in hepato-biliary surgery, transplant surgery and segmental transplantation. For adults the best option for an appropriate graft is the right hepatic lobe. Today many centers are using the right hepatectomy in living donor liver transplantation. Only Asian countries utilize the left lobe, but just for smaller adult recipients. The left lobe graft or the left lateral segments are used currently in pediatric patients. Therefore, it is of paramount importance to know exactly the liver anatomy and the variations of the hepatic arteries, veins and biliary tree. The plane of transection used for obtaining the right liver graft is indicated by the middle hepatic vein. This vein remains in most of the cases in the donor remnant along with the main hepatic artery, portal vein and hepatic duct. The right lobe graft contains segments 5, 6, 7 and 8 .

The right hepatic vein drains the right lobe segments. Sometimes it may drain portions of segment 4 . The middle hepatic vein drains segment 4 , the medial part of the left lobe and sometimes major branches extend to the right, to drain the right paramedian sector. In many centers the section plane is placed to the right of the middle hepatic vein. However, venous variants are common. Seventeen percent of the population has 2 or more hepatic veins which drain the right lobe, while 10\% have an accessory inferior hepatic vein. Also frequently major branches of the middle hepatic vein drain segments 5 and 8. If they are larger than $5 \mathrm{~mm}$, instead of ligating, they are reconstructed in the recipient to prevent congestion of the graft by inadequate venous outflow. The donor operation is performed without inflow occlusion and the venous central pressure is maintained low $(3-5 \mathrm{mmHg})$ to prevent bleeding. The use of cell-saver is helpful. After cholecystectomy the right hepatic artery and the portal vein bifurcation are isolated with no dissection on the left side of the hepato-duodenal ligament. It is important for the safety of the donor to not hurt the left hepatic artery and to spare large branches for segment 4 . The retrohepatic veins are individualized after mobilization of the right lobe and dissection of the right triangular and coronary ligaments. These veins are either ligated or resected and further re-implanted if they are larger than $5 \mathrm{~mm}$. No dissection of the left triangular ligament is done to avoid a later torsion of the remnant left lobe. The anatomy of the bile duct is verified with a probe through the cystic duct or minicholedochotomy, and the right bile duct is transected [6]. We have to keep in mind that the biliary anatomy is variable. The magnetic resonance cholangiography or the all-in-one CT with software that reconstructs in $3 \mathrm{D}$ the vascular anatomy and the biliary tree are of great help, as it is important to know the exact anatomy of the biliary tree, because the biliary anastomosis in the recipient is considered to be the "Achilles heel" of the procedure. Preoperative magnetic resonance cholangiography is useful, but is not a substitute for intraoperative cholangiography. The line of resection is the Cantlie line, visible after a hemi-Pringle maneuver and an ultrasound evaluation of the middle hepatic vein. The transection performed with the ultrasonic dissector is made without occlusion of the vascular components. The middle hepatic vein stays usually with the donor [6]. The major branches of the middle hepatic vein (segment 5 and 8) are resected for future reconstruction. It is very important to avoid the congestion of the right antero-medial sector of the graft. After parenchymal transection, the right lobe is still attached to the donor through its vascular structures. Hemostasis is checked on the hepatic raw surfaces and after the completion of the recipient hepatectomy the right lobe graft is removed from the donor, while the left liver lobe remains in place. The graft is flushed after explantation with cold "University of Wisconsin" solution. When venous reconstruction is needed, one can use on the back table venous or arterial allografts (jump grafts). The biliary tree has to be flushed in order to prevent caustic damage of the epithelium during the cold ischemia. The graft hepatic artery is sutured to the proper, right or left hepatic artery of the recipient. Due to the larger caliber of the right ar- 
tery, thrombosis may be uncommon in living donor liver transplantation.

In the recipient a temporary porto-caval shunt is used in certain situations. The right hepatic vein is anastomosed to the recipient's right hepatic vein, or to enlarged openings at the same site. Large inferior hepatic veins are connected directly to the cava vein. To avoid congestion of the anteromedial sector and to enhance the outflow, venous reconstruction may be needed. The congestion is documented by hepatofugal flow in the portal vein and by discoloration of the antero-medial sector after arterial clamping [7]. The graft portal vein is connected to the main portal vein of the recipient. The graft hepatic artery is sutured to the proper, right or left hepatic artery. The bile duct is reconstructed with a Roux-en-Y loop or endto-end or end-to-side anastomosis with T-tube drainage. The stenting of the biliary anastomosis decreases the occurrence of complications.

\section{Discussion}

The living donor liver transplantation is a procedure performed in highly qualified centers as an option to the shortage of cadaveric organs, in an attempt to decrease the number of patients on the waiting list. For adult patients the best option is a right lobe graft from the living donor. The indications are end stage liver diseases like cirrhosis, liver cancer and acute liver failure. Mortality risk for the donor is estimated to be $0.2-0.4 \%$.

The operation was introduced on the principle that today, right and left hepatectomies are performed with almost no mortality. Until the living donor liver transplantation (especially with right liver lobes), the hepatic transplant teams focused on the inflow (the arterial anastomoses) because of the risk of hepatic artery thrombosis and acute complications. The loss of several grafts without presenting any arterial dysfunction lead to the idea that the venous obstruction and congestion of the right paramedian sector can cause the liver failure. It is known also that venous congestion may interfere negatively with the biliary anastomoses. Previous ligation of the major hepatic veins has no risk for patients undergoing hepatectomies, due to collateral pathways via the sinusoids and short hepatic veins.

The most notable controversy in the donor operation refers to the middle hepatic vein and evaluation of the hepatic congestion of the graft. Regurgitation in the portal flow measured by Doppler ultrasound indicates a congested area in the liver. The portal vein becomes the draining vein and there is no venous outflow from that area, meaning a nonfunctional hepatic zone. Another method to indicate hepatic congestion in the right paramedian sector is temporary arterial clamping when the veno-occlusive area appears discolored. If the volume of the graft after extracting the congested zone is estimated to be not sufficient to support the metabolic demands, a venous reconstruction (using the hepatic vein or its tributaries) is mandatory [8].
Many efforts have been made to improve the venous outflow of the grafted right lobe. Marcos [8] recommends a plane of hepatic transection which includes the distal part of the middle hepatic vein and large branches from segment 8 and 5 . Also inferior hepatic veins larger than 5 $\mathrm{mm}$ are sutured to the vena cava. The venous reconstruction aims to improve the outflow with a large anastomosis with or without a jump graft. Some transplant centers are using grafts including the entire middle hepatic vein, but there are some concerns from others regarding the safety of the donor [9]. These right extended lobes are 10\% larger than the standard right grafts, but $70 \%$ of the hepatic donor mass is removed, only $30 \%$ remaining with the donor [10]. Also a remnant left lobe in the donor without the middle hepatic vein can cause congestion and malfunction of segment 4. As a result, even when harvesting the middle hepatic vein, if a large branch fromsegment 4 is encountered, the division of the middle hepatic vein should be done proximally in order to avoid the obstruction of the venous drainage of this segment [11]. Also an umbilical vein present in $70 \%$ of cases helps to drain segment 4 . Regardless of our option concerning the middle hepatic vein, the safety of the donorremains the most important issue $[12,13]$, being mandatory to ensure that the remnant lobe will sustain the metabolic demands after surgery.

\section{Conclusions}

Living donor liver transplantation has emerged as a response to the scarcity of cadaveric organs in an effort to increase the donor pool. The goal of the new technique described in this review was to decrease the number of patients on the waiting list for liver transplantation. This procedure was first introduced for pediatric patients who received the left lateral hepatic segments from the donor. Subsequently the right liver lobe was harvested from adult living donors and used in adult patients. This procedure has several advantages: surgery performed electively, superior quality of the grafts compared to cadaveric ones and it gives a chance to patients who are not eligible for cadaveric organ transplantation. The disadvantage is represented by the potential risk to the donor, who is a healthy person. Worldwide at least 6 donor deaths were reported, the mortality of the procedure being between $0.2-0.4 \%$. It is obvious that donor safety is the key of the living donor liver transplantation procedure. This can be achieved by assessing the complex anatomy of the liver, possessing skills in hepato-biliary surgery and following current guidelines. With improved technology, the procedure will be safer for the donor and the graft will function better in the recipient. Also the surgeons have to be well trained and experienced in these techniques, which are performed in highly specialized transplant centers.

\section{References}

1. Broelsch CE, Frilling A, Nadalin S, et al. Leberlebendspende. Die deutsche Erfahrungim international Vergleich. Chirurg. 2003;74(6):510-522. 
2. Shiffman ML, Brown RS Jr, Olthoff KM, et al. Lving donor liver transplantation: Summary of a conference at the National Institutes of Health. Liver Transplantation. 2002;8(2):174-188.

3. Malago M, Testa G, Frilling A, et al. Right living donor liver transplantation: An Option for Adult Patients. Annals of Surgery. 2003;238(6):853-863.

4. Marcos A. Right lobe living donor liver transplantation: A Review. Liver Transplantation. 2000;6(1):3-20.

5. Urata K, Kawasaki S, Matsunami H, et al. Calculation of child and adult standard liver volume for liver transplantation. Hepatology. 1995;21:13171321.

6. Malago M, Testa G, Marcos A. Ethical considerations and rationale of adult-to-adult living donor liver transplantation. Liver Transplantation. 2001;7(10):921-927.

7. Sano K, Makuuchi M, Miki K, et al. Takayama. Evaluation of hepatic venous congestion: Proposed indication criteria for hepatic vein reconstruction. Annals Of Surgery. 2002;236(2):241-247.
8. Marcos A, Orloff M, Mieles L, et al. Functional venous anatomy for rightlobe grafting and techniques to optimize outflow. Liver Transplantation. 2001;7(10):845-852

9. Fan ST, Lo CM, Liu CL. Safety and necessity of including the middle hepatic vein in the rightlLobe graft in adult-to-adult live donor liver transplantation. Annals of Surgery. 2003;328(1):137-147.

10. Sugawara $Y$, Makuuchi M, Sano K. Vein reconstruction in modified right liver graft for living donor liver transplantation. Annals Of Surgery. 2003;237(2):180-185.

11. Ghobrial RM, Hsieh CB, Lerner Susa, et al. Technical challenges of hepatic venous outflow in right lobe adult living donor liver transplantation. Liver Transplantation. 2001;7(6):551-555.

12. Makuuchi M, Miller CM, Olthoff $\mathrm{K}$, et al. Adult-adult living donor liver transplantation. Journal of Gastrointestinal Surgery. 2004;8(3):303-312.

13. Abecassis M, Koffron A, Fryer J, et al. Surgical techniques in living donors. Current Opinion in Organ Transplantation. 2001;6:355-358 